\title{
Automatic Registration of Satellite Images
}

\author{
Ritu Singh Phogat \\ M.E. Student \\ MEFGI, Rajkot
Bharat Chaudhary
(Project Scientist) \\ BISAG, Gandhinagar
}

\author{
Hardik Dhamecha \\ Asst.Prof. \\ MEFGI, Rajkot
}

\author{
Manoj Pandya, PhD \\ (Project Manager) \\ BISAG, Gandhinagar
}

\author{
Madhukar Potdar, PhD \\ (Project Director) \\ BISAG, Gandhinagar
}

\begin{abstract}
Registration is a fundamental stage in $3 \mathrm{D}$ and $2 \mathrm{D}$ reconstruction process, which is used to match two or more images that can be taken in different moments, from different sensors, or different viewpoints. Two images are aligned into a common coordinate system in order to monitor the subtle changes between them. Registration algorithms compute transformations to set correspondence between the two images. . This approach provides a good basis for classifying different techniques, however it does little to illuminate the conditions important to the problem of registration. Understanding the image registration problem space and the conditions under which specific algorithms perform best allows for further automation of image registration, and makes the field more accessible to non-experts. This paper presents manual and automatic registration and their comparison is made.
\end{abstract}

Keywords: Manual Registration, Automatic Registration, Rectification

\section{INTRODUCTION}

In image processing, for example, when combining the information content of image, we are interested in the relationship between two or more images. The analysis of this relationship usually be easily controlled or dealt with once a correspondence is set up between the images. The task of setting up this correspondence is called image registration. Image registration is basically of two types:

A. Image to image registration: Here two or more images are aligned to integrate or fuse corresponding pixels that represent the same objects.

B. Image to map registration: Here input image is warped to match the map information of a base image, while retaining the original spatial resolution. Image registration is the process of calculating spatial transforms which align a set of images to a common observational frame of reference, often one of the images in the set. Registration is a key step in any image analysis or understanding task where different sources of data must be combined. It is a critical component of computational photography [1] remote sensing [2], [3], automatic manufacturing processes, and medical image processing [4], [5].More recently it has been used to create navigable models of a scene from a database of photographs [6], to remove unwanted objects from overlapping images, etc. During registration process two situations become evident. First, it is impossible to determine which of the coordinate system points of one image matches with the corresponding points of another image, this is known as matching problem, and is also most time consuming stage during the execution of algorithm. Second, there is need of transformation in the three dimensional information of one of the images regarding its coordinate system and its relationship to the image that was chosen as its reference. In simple words, image registration process is an automatic or manual procedure which tries to find corresponding points between two images and align them spatially to minimize a desired error, that is a consistent distance measure between two images. There is a rapid development in image acquisition devices during last few decades and also growing amount and diversity of images that are obtained has invoked the research on automatic image registration. The intention of this article to provide information manual and automatic registration and their comparison

\section{MANUAL IMAGE REGISTRATION}

Here satellite image taken is a tiff image of very large size of about $274 \mathrm{MB}$ for image to map registration and stereo images for image to image registration. For registration, along with satellite images metadata is also used. For manual registration ENVI software was used in which ground control points were selected manually from the given metadata and were placed at four corners in the tiff image. The following figure shows the algorithm or the flow chart of manual image to map registration.

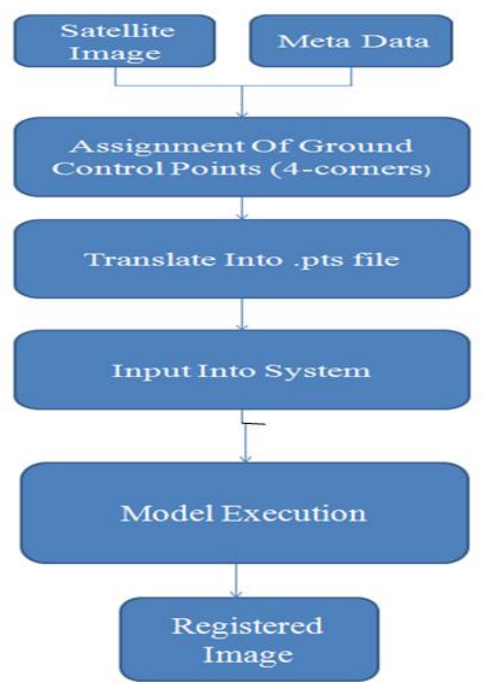

Fig: 1 Manual Registration 
Satellite image is first displayed on screen, After this, the ground control points (values of latitude and longitude) is selected and placed on an image. This file is converted into .pts file in which the values of GCPs are stored in text format and can be used again if registration is performed again. So that there is no need to select GCP again and again. While doing image to map registration next time this .pts can be used to display these values. Since only four GCPs are selected so there is no error, otherwise error occur when fifth GCP is selected.

After selection of GCPs, this image is warped on map.

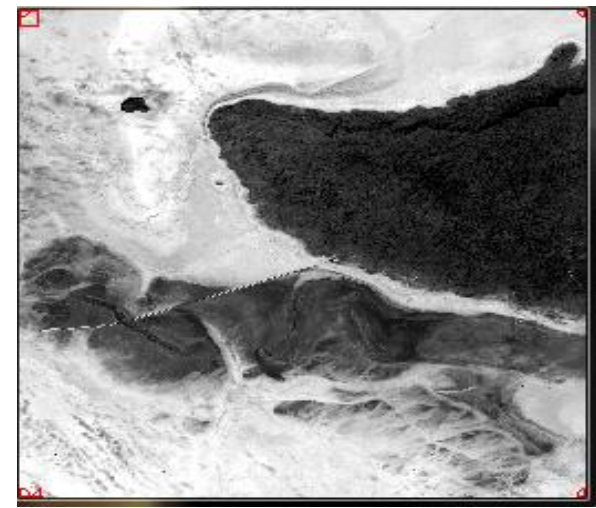

Fig: 2 GCP at corners

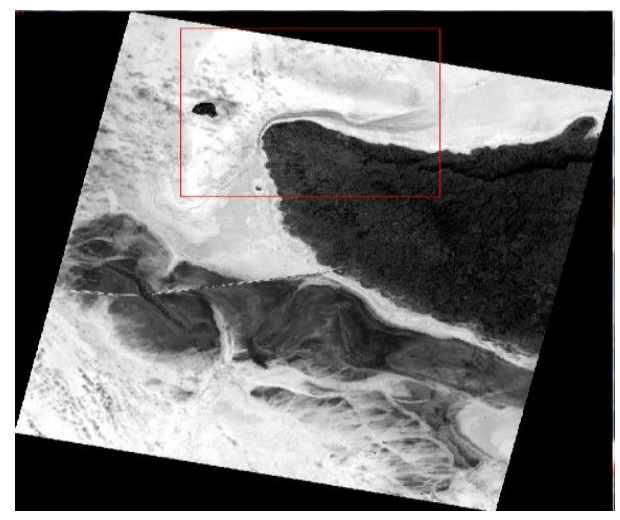

Fig: 3 Image to map registration

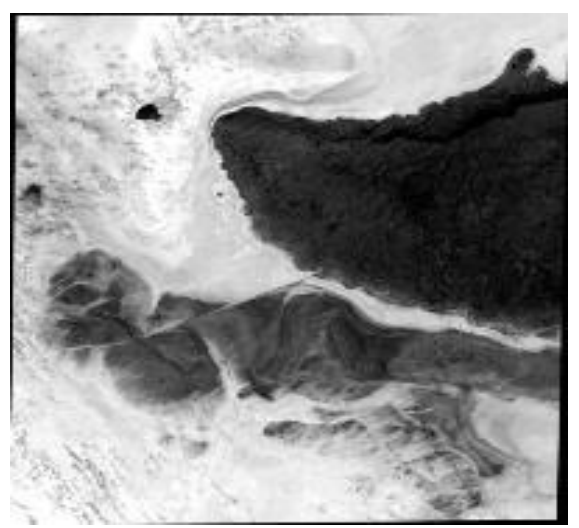

Fig: 4 Image to image registration

\section{AUTOMATIC IMAGE REGISTRATION}

Image stereo-rectification is the process by which two images of the same solid scene undergo homographic transforms, so that their corresponding epipolar lines coincide and become parallel to the $\mathrm{x}$-axis of image. A pair of stereo-rectified images is helpful for dense stereo matching algorithms. In this paper robust geographic image rectification is done where Horris corners are selected and correspondence is made between them using RANSAC algorithm. The precise 3D reconstruction task requires an accurate dense disparity map, which is obtained by image registration algorithms. By estimating the epipolar geometry between two images and performing stereo-rectification, the search domain for registration algorithms is reduced and the comparison simplified, because horizontal lines with the same y component in both images are in one to one correspondence. Stereo-rectification methods simulate rotations of the cameras to generate two coplanar image planes that are in addition parallel to the baseline. The following figure shows the algorithm of robust image rectification.

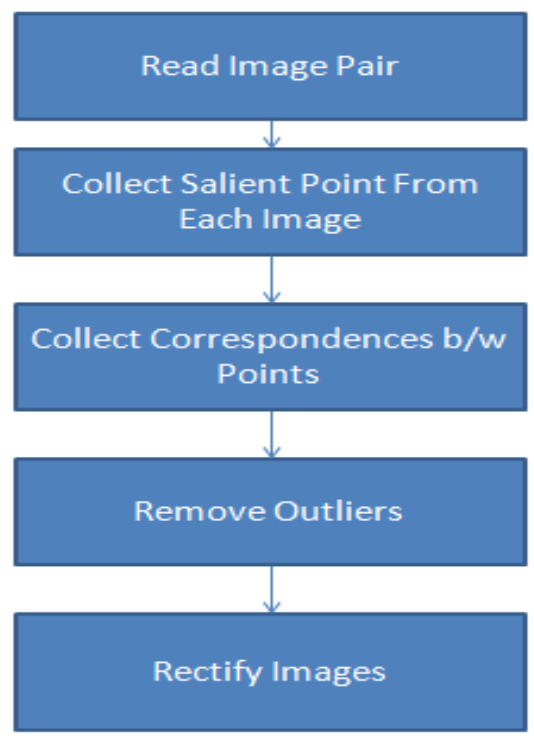

Fig: 5 Algorithm of image rectification 


\subsection{Implementation and Results}

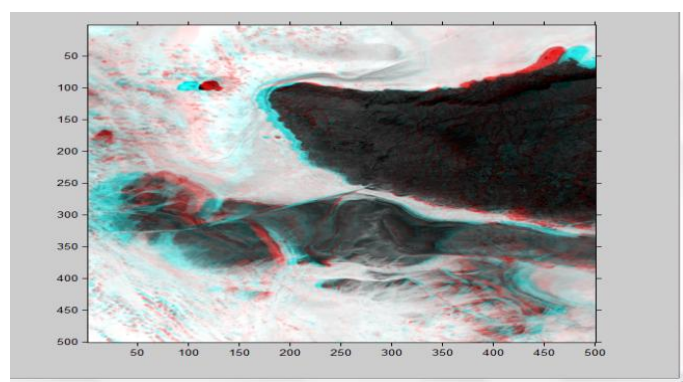

(a)

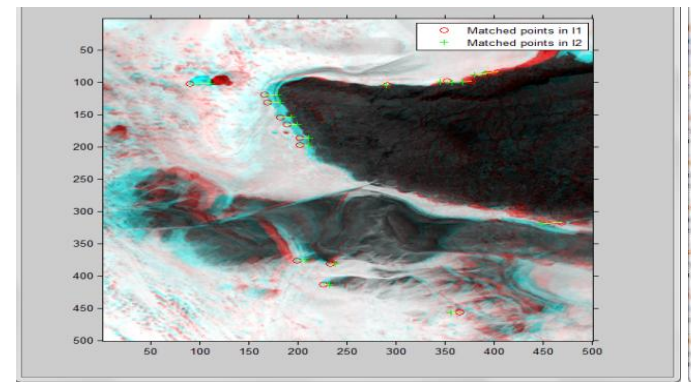

(c)

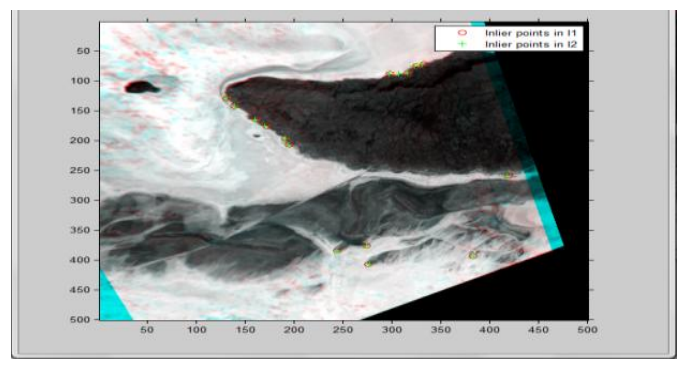

(e)

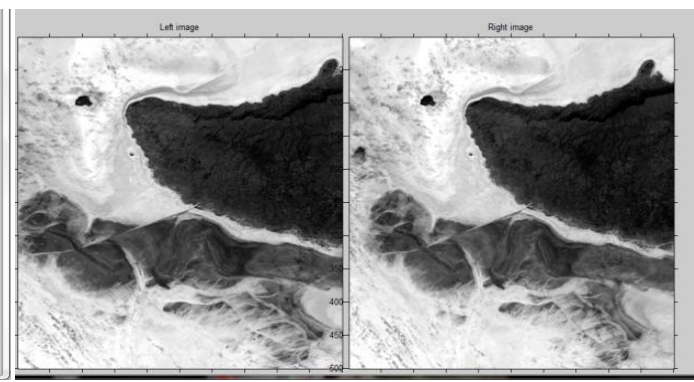

(b)

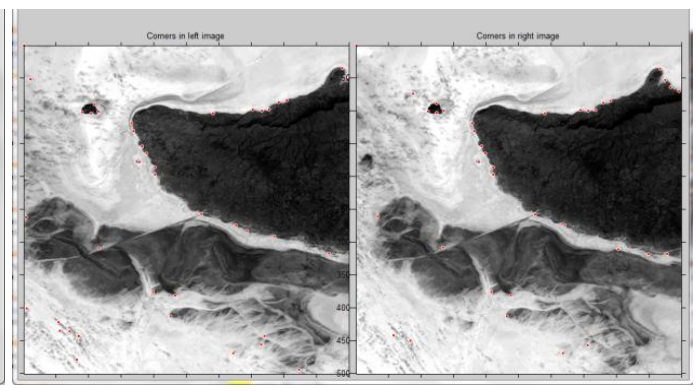

(d)

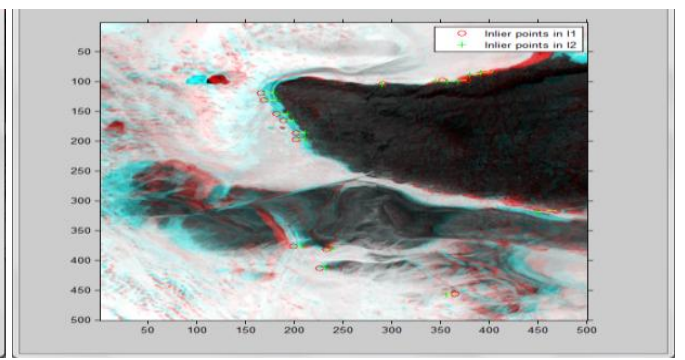

(f)

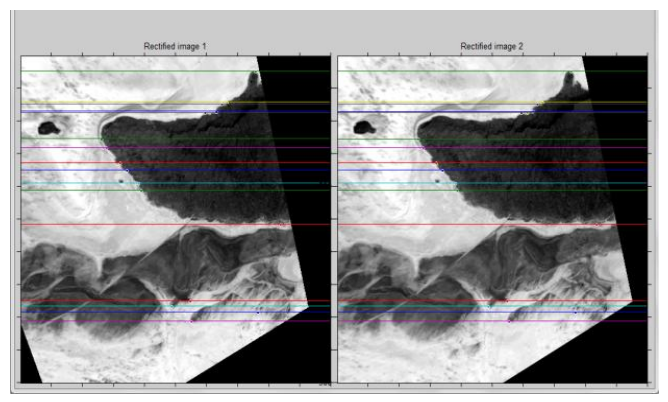

(g)

Fig: 6 (a) stereo images (b) colour composite (c) corners selected (d) correspondence created (e) removed bad outliers (f) correspondence on same row (g) rectified image

\section{MANUAL V/S AUTOMATIC REGISTRATION RESULTS}

Due to earth's curvature and satellite's continous movement there is difference in images of same scene taken in terms of resolutions, zoom scales, and gray levels, so on. After registration there is transformation of $3 \mathrm{D}$ scene in $2 \mathrm{D}$ image because of which geometric error is created. 


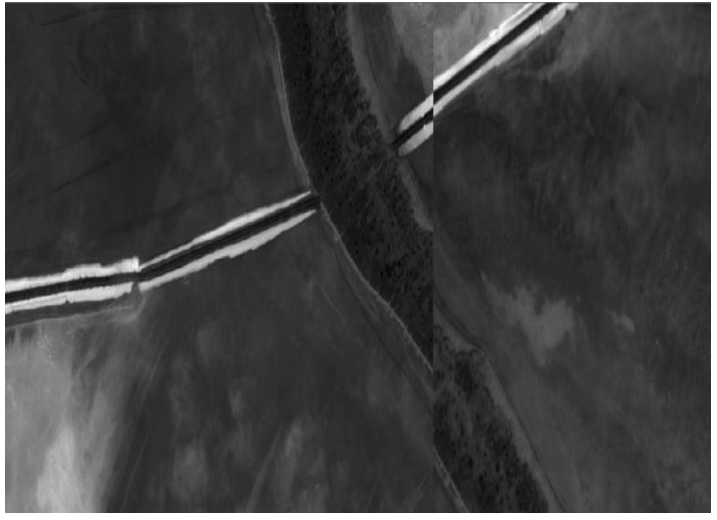

Fig: 7 Geometric Error

\begin{tabular}{|c|c|c|c|c|}
\hline & Base X & Base $Y$ & Warp X & Warp Y \\
\hline$\# 1+$ & 4045.00 & 2987.00 & 4373.00 & 2659.00 \\
\hline$\# 2+$ & 4373.00 & 3784.00 & 4607.00 & 3691.00 \\
\hline$\# 3+$ & 5076.00 & 5191.00 & 5264.00 & 5050.00 \\
\hline$\# 4$ + & 7232.00 & 6316.00 & 6857.00 & 6269.00 \\
\hline$\# 5+$ & 8920.00 & 6879.00 & 8404.00 & 6925.00 \\
\hline$\# 6+$ & 7045.00 & 2426.00 & 7232.00 & 2472.00 \\
\hline$\# 7+$ & 11732.00 & 832.00 & 10982.00 & 925.00 \\
\hline$\# 8+$ & 9389.00 & 2098.00 & 9107.00 & 2237.00 \\
\hline$\# 9+$ & 10935.00 & 7395.00 & 11123.00 & 7581.00 \\
\hline$\# 10$ - & 4842.00 & 4395.00 & 4935.00 & 4347.00 \\
\hline
\end{tabular}

Fig: 8 List of Ground Control Points Selected

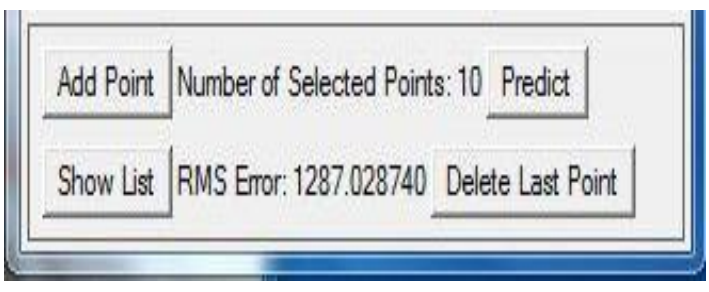

(h)

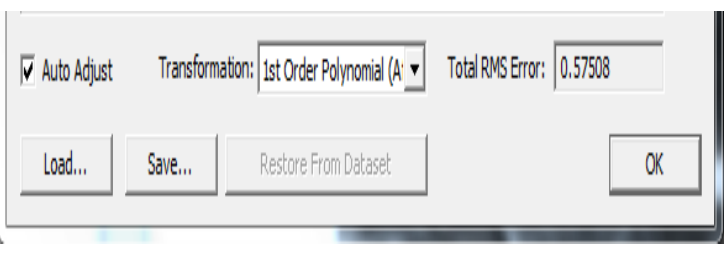

(i)

Fig: 9 (h) Manual Registration Error (i) Automatic Registration Error

After performing automatic registration the format of final image was .fig which was not compatible with georeferencing softwares like ENVI, QGIS and many more. So it was converted to jpeg format and a jgw file was created manually. Thus the image was georeferenced in QGIS software and was found that there is tremendous reduction in error value compare to manual registration. This is shown in the above figure. During performance of manual and automatic registration 10-12 points were selected and vast difference in error value was found. As shown in fig 9 the RMS error for manual registration is about 1287 while after performing automatic registration it has been reduced to about 0.575 which is a very big difference. The reason of this difference is that during manual registration the GCPs are not selected very accurately, whereas in automation process harris corners are selected very accurately which results in proper selection of GCPs as a result of which less error is obtained.

\section{CONCLUSION AND FUTURE SCOPE}

In this research paper algorithm of manual registration, its implementation and results are shown. Also the automatic registration, its implementation and results are shown. 10-12 GCPs are selected and some error is obtained in both the processes. At last, comparison is made between them and it is found that automatic process gives much better and robust result compare to manual registration. Image registration is difficult when images are obtained through different sensor types. It is observed that method based on information theory yields a more accurate registration than any other registration method. An automatic matching method cannot be used for matching of control points as the deformation between images is arbitrary [7]. In the future, we need to develop an expert image registration method from a combination of various approaches which takes care of the type of the given task and provides an appropriate solution.

\section{ACKNOWLEDGEMENT}

The authors wish to thank T.P. Singh, Director, Bhaskaracharya Institute of Space Application and GeoInformatics for his valuable support and inputs in the research work.

\section{REFERENCES}

[1] A. Agarwala, M. Dontcheva, M. Agrawala, S. Drucker, A. Colburn, B. Curless, D. Salesin, and M. Cohen, "Interactive digital photomontage," in SIGGRAPH '04: ACMSIGGRAPH 2004 Papers, New York, NY, USA, 2004, pp. 294-302.

[2] T. M. Lillesand and R. W. Kiefer, Remote Sensing and ImageInterpretation, 6th ed. Wiley, 2007

[3] J. B. Campbell, Introduction to remote sensing, 4th ed. Guildford Press, 2008.

[4] J. Maintz and M. Viergever, "A survey of medical image registration”, Medical Image Analysis, vol. 2, no. 1, pp. $1-36,1998$

[5] J. Pluim, J. Maintz, and M. Viergever, "Mutualinformationbased registration of medical images: a survey",Medical Imaging, IEEE Transactions on, vol. 22, no. 8, pp. 986-1004, Aug. 2003. 313

[6] Pascal, Jean-Michel, Zhongwei, "Three-step image rectification" BMVC doi:10.5244/C.24.89,2010

[7] B. Zitova and J. Flusser, "Image registration methods: a survey," Image and Vision Computing, vol. 21, pp. 977 1000, 2003.

[8] A. C. Morris, A. Stevens, J.P. Muller," Ground control determination for registration of satellite imagery using digital map data". Photogram Record, 1998.

[9] S. Kullback, Information theory and statistics. Dover Publications, Inc., 1968.

[10] T.M. Cover and J.A. Thomas, Elements of information theory, John Wiley \& Sons, Inc., 1991 